\title{
Ultra-thin Skin Electronics for High Quality and Continuous Skin-Sensor-Silicon Interfacing
}

\author{
Leilai Shao $†$, Sicheng Li†, Ting Lei†, Tsung-Ching Huang, Raymond Beausoleil, Zhenan Bao, and \\ Kwang-Ting Cheng
}

\begin{abstract}
Skin-inspired electronics emerges as a new paradigm due to the increasing demands for conformable and high-quality skin-sensorsilicon (SSS) interfacing in wearable, electronic skin and health monitoring applications. Advances in ultra-thin, flexible, stretchable and conformable materials have made skin electronics feasible. In this paper, we prototyped an active electrode (with a thickness $\leq 2 \mathrm{um}$ ), which integrates the electrode with a thin-film transistor (TFT) based amplifier, to effectively suppress motion artifacts. The fabricated ultra-thin amplifier can achieve a gain of $32 \mathrm{~dB}$ at 20 $\mathrm{kHz}$, demonstrating the feasibility of the proposed active electrode. Using atrial fibrillation (AF) detection for electrocardiogram (ECG) as an application driver, we further develop a simulation framework taking into account all elements including the skin, the sensor, the amplifier and the silicon chip. Systematic and quantitative simulation results indicate that the proposed active electrode can effectively improve the signal quality under motion noises (achieving $\geq 30 \mathrm{~dB}$ improvement in signal-to-noise ratio (SNR)), which boosts classification accuracy by more than $19 \%$ for AF detection.
\end{abstract}

\section{INTRODUCTION}

Emerging applications, from wearable, bio-medical therapy, disease prevention to electronic-skin, require flexible electronics to provide continuous, long-term and high quality human-machine interfaces with great comfortness and wearability for the users. Among flexible electronic materials, the polymers, carbon nanotubes, nano-wires and nano-crystal show great potentialsfor skininspired electronics [1-3] . Skin electronics, which involves flexible materials and device integration to enable skin-like properties, has enabled many desirable features for wearable applications such as ultra-thin form factor, mechanical flexibility, stretchability and conformable adhesion to the human body $[4,5]$. Among various emerging flexible materials, carbon nanotube (CNT) shows great potentials for high-performance skin electronics due to its high carrier mobility, mechanical flexibility, and low-cost manufacturing [6]. In Fig. 1a-b, we designed and fabricated ultra-thin $(<2 \mu m) \mathrm{CNT}$ circuits along with 3D illustration of the device's layer structure, where CNT thin-film transistor (TFT) circuits are fabricated on a $1 \mu \mathrm{m}$ polymer substrate. As shown in Fig. 1b, electrodes, interconnects, barrier, CNT and encapsulation layers are deposited in the

$\dagger$ These authors contribute equally in this work. L. Shao, is with the Department of Electrical and Computer Engineering, University of California Santa Barbara, CA 93106, USA. E-mail: 1shao@ece.ucsb.edu. L. Ting and Z. Bao are with Department of Chemical Engineering, Stanford University, Stanford, California 94305, USA. T-C. Huang, S. Li and R. Beausoleil are with Hewlett Packard Labs, Palo Alto, California 94305, USA. K-T. Cheng is with School of Engineering, Hong Kong University of Science and Technology, Hong Kong, China. The developed compact model and simulation framework is available at: https://github.com/llshao/CNT-TFT-Verilog-A

Permission to make digital or hard copies of all or part of this work for personal or classroom use is granted without fee provided that copies are not made or distributed for profit or commercial advantage and that copies bear this notice and the full citation on the first page. Copyrights for components of this work owned by others than the author(s) must be honored. Abstracting with credit is permitted. To copy otherwise, or republish, to post on servers or to redistribute to lists, requires prior specific permission and/or a fee. Request permissions from permissions@acm.org.

DAC '19, June 2-6, 2019, Las Vegas, NV, USA

(C) 2019 Copyright held by the owner/author(s). Publication rights licensed to ACM.

ACM ISBN 978-1-4503-6725-7/19/06 ..\$15.00

https://doi.org/10.1145/3316781.3317928

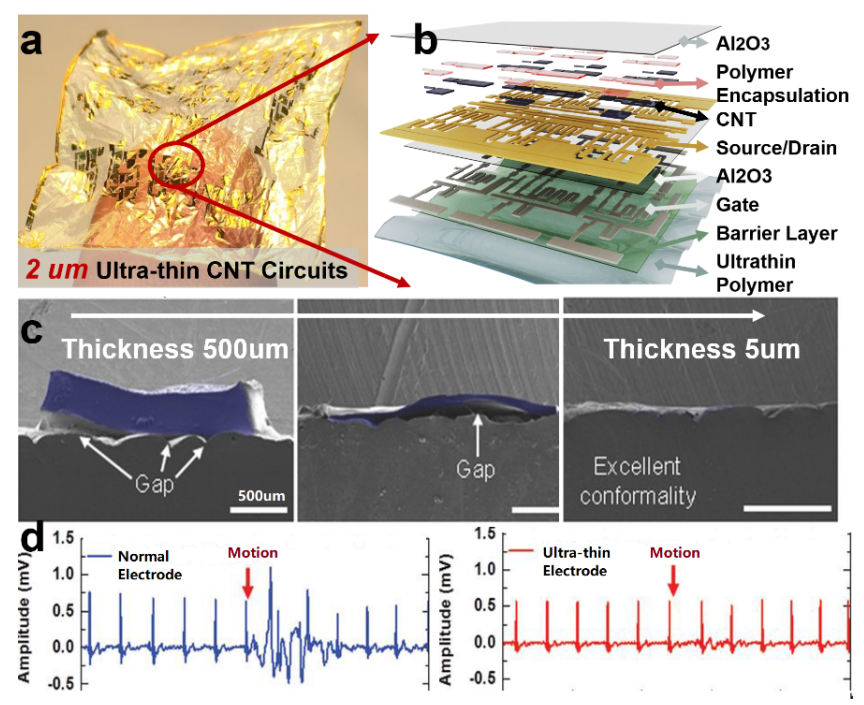

Figure 1: a\&b. Ultra-thin flexible CNT TFT circuits [7] ; c. ultra-thin electronics enabled conformable contacts; $d$. motion suppression with ultra-thin electrodes. Reproduced with permission $[4,5]$. Copyright 2013, 2014, Wiley .

illustrated order. In Fig. 1c, the scanning electron microscope (SEM) of various electrode on artificial skin shows that a conformable contact can be achieved with the ultra-thin circuits $(<5 \mu m)$ [4]. Such a conformable contact brings not only better wearability but also effective suppression of motion induced artifacts for bio-signals detection [5]. As illustrated in Fig. 1d, both normal and ultra-thin electrodes are placed on the same place to record the ECG signal, when the subject moves, indicated by the red arrow, a significant motion artifact appears for a traditional electrode while an ultra-thin conformable contact electrode effectively suppresses the motion noise. Therefore ultra-thin $(<2 \mu \mathrm{m})$ CNT-TFT circuits are highly desirable for the high quality and long-term skin-sensor-silicon (SSS) interfacing for continuous health-monitoring applications.

Previous studies of skin electronics mainly focus on material and device level innovations $[8,9]$. In this paper, we demonstrate a skinsensor-silicon interfacing as illustrated in Fig. 3 and offer systematic and quantitative analysis for the demonstration. The main focus of the SSS interface is on detecting and improving signal quality for wearable applications. We first demonstrate how an ultra-thin electrode can effectively reduce the motion-induced noise and we use an electrocardiogram (ECG) recording system as the use case. After identifying the noise sources in an SSS link, we further propose the ultra-thin active electrode, which integrates an ultra-thin CNT-TFT amplifier with the sensor/electrode as shown in Fig. 3b. The ultra-thin amplifier provides low-noise signal amplification for the signal-of-interest (SOI), is fully integrated with the ultrathin electrodes, and thus greatly boosts the signal integrity. The signal quality improvement cannot be achieved by the typical configuration consisting of thick electrodes and rigid silicon amplifier located in a thick silicon chip (far away from the sensor/electrode), 
where noises have already entangled with the SOI in the interconnects/interfacing as indicated in Fig. 3. Heavy signal processing methods have been proposed to improve the signal quality $[10,11]$; however, high power consumption $(>10 \mathrm{~mW})$ makes them not suitable for continuous wearable applications. Customized ASIC can achieve $<1 \mathrm{~mW}$ power consumption [12], but its high cost (when in low volume production) and relatively long developing periods limit its broad adoption for a wide range of applications.

In this study, we designed and fabricated an ultra-thin active electrode with an integrated ultra-thin CNT-TFT amplifier, successfully achieving a voltage gain of $32 \mathrm{~dB}$ running at $\sim 20 \mathrm{kHz}$, shown in Fig. 4. To quantify the improvement of signal quality achieved by the proposed active electrode, we develop an end-to-end simulation framework for an SSS link including the skin, the sensor, the amplifier, interconnects and a classifier for atrial fibrillation (AF) detection, shown in Fig. 5. The framework enables analysis of how front-end SSS design affects the overall system performance, such as the classification accuracy for AF detection. The main contributions of this paper are summarized as follows:

- Proposed an ultra-thin active electrode which integrates an ultra-thin flexible amplifier with the electrode, opening a brand new design space for skin-sensor-silicon interfaces.

- Designed and prototyped an ultra-thin CNT-TFT amplifier with a gain as high as $32 \mathrm{~dB}$ running at $\sim 20 \mathrm{kHz}$, which provides clear evidence of the feasibility of the proposed active electrode design.

- Developed an end-to-end simulation framework which takes into account all relevant elements including the skin-sensorsilicon interface and the machine learning classifier, enabling systematic exploration and analysis of the impacts of frontend SSS interface on the overall classification accuracy.

- Quantitatively evaluated how the proposed active electrode can improve the signal quality under motion noises (achieving $>35 \mathrm{~dB}$ signal-to-noise ratio (SNR) boost) and how the front-end SSS design affects the overall classification accuracy (boosted from $65.5 \%$ to $84.6 \%$ ).

The rest of this paper is organized as follows: Section 2 introduces the SSS interface and our prototyped active electrode. Section 3 elaborates the end-to-end simulation framework including skin, sensor, amplifier and a deep-learning based classifier. Quantitative experimental results are described in Section 4. Section 5 discusses possible directions for future improvements. Section 6 draws some conclusion.

\section{SKIN-SENSOR-SILICON INTERFACING}

A high quality skin-sensor-silicon (SSS) interface is critical for acquiring high-quality biological signals, while traditional $\mathrm{Ag} / \mathrm{AgCl}$ electrodes with wet conductive gels could not ensure comfortable user experience particularly for continuous monitoring. Dry electrodes are more comfort to wear, however, they are more vulnerable to motion artifacts and interconnects noise $[13,14]$. In contrast, ultra-thin skin electronics provides a promising solution to high quality interfacing suitable for long-term monitoring.

\subsection{Noise Suppression via Near Sensor Amplification}

In an electrocardiogram (ECG) recording system, there are mainly three noise sources: baseline wander (BW), electromyography (EMG) and motion artifacts/interference [11]. The noise signals used in this study are from the PhysioNet MIT-BIH noise stress database [15]. Typical samples of these three noises, in both time and frequency domain, are shown in Fig. 2. As illustrated, the BW noise lies mainly in the low frequency domain $(<0.5 \mathrm{~Hz})$, which can be filtered out using a high pass filter. EMG and motion noises are relatively
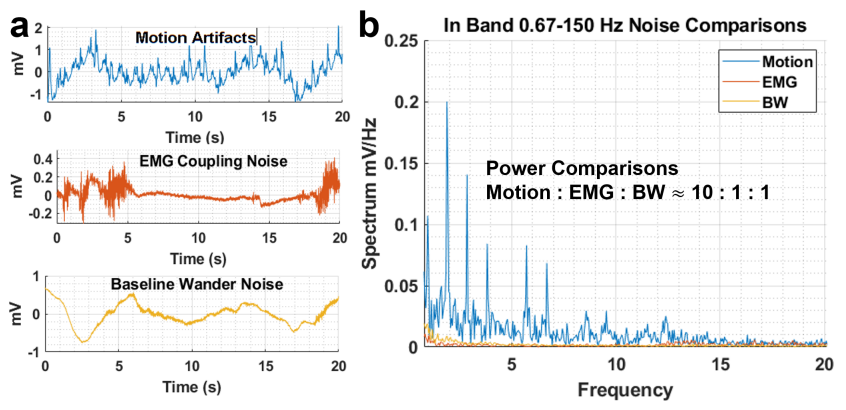

Figure 2: Three main noises for ECG recording system; a. waveforms of different noises; b. spectrum analysis (only 0 $20 \mathrm{~Hz}$ is shown here) and power comparisons (integral over 0.67-150 Hz).

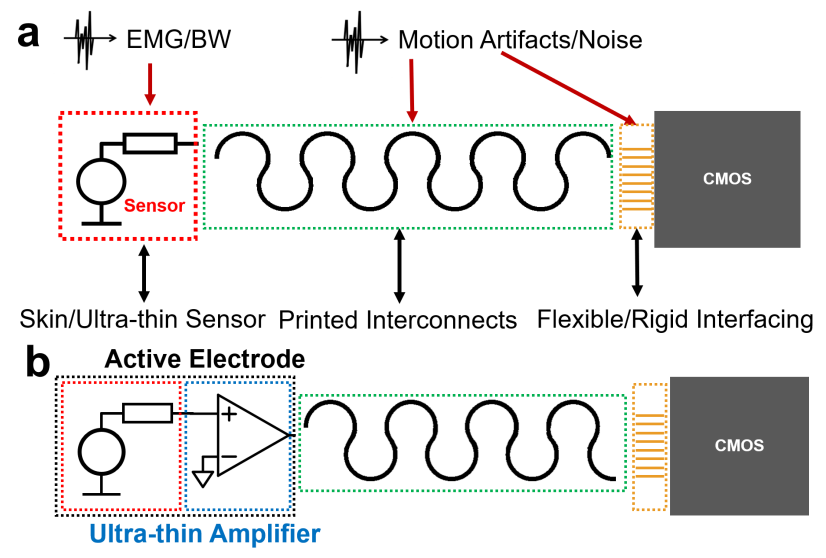

Figure 3: a. Illustration of a Skin-Sensor-Silicon interface; b. proposed active electrode by integrating ultra-thin amplifiers with the electrode.

widespread over $0-500 \mathrm{~Hz}$. Our driving application in this study is atrial fibrillation (AF) detection, whose useful information mainly lies within $0.67-150 \mathrm{~Hz}$ [16]. Thus, we compared these three noises' power in the band of $0.67-150 \mathrm{~Hz}$. The motion noise turns out to be the most significant noise source (Motion:EMG:BW $\approx 10: 1: 1$ ), for which an ultra-thin skin electronics could effectively overcome such motion artifacts.

As presented in Fig. 3a, the motion artifacts can be suppressed with an ultra-thin electrode which also offers conformable contacts with skin; however, the subsequent parts, such as interconnects and flexible/rigid interfacing, are still vulnerable to the motion noise. To further improve the signal quality for such an SSS interfacing, we propose a novel active electrode design, which integrates the electrode with an CNT-TFT ultra-thin amplifier to effectively suppress motion artifacts and interconnects' interference. This idea is demonstrated in Fig. 3b, where signal-of-interest (SOI) is pre-amplified by the ultra-thin integrated amplifier. For study its feasibility, we designed and prototyped an ultra-thin CNT-TFT based amplifier, which will be elaborated in the following subsection.

\subsection{Active electrode for the SSS Interfacing}

Most previous reported TFT based amplifiers are thicker than 50 $\mu m$ [17-19], which cannot meet the stringent requirement of conformability for seamless contacts. The organic TFT based amplifier reported in [17] achieves a thickness of less than $5 \mu \mathrm{m}$; however, 

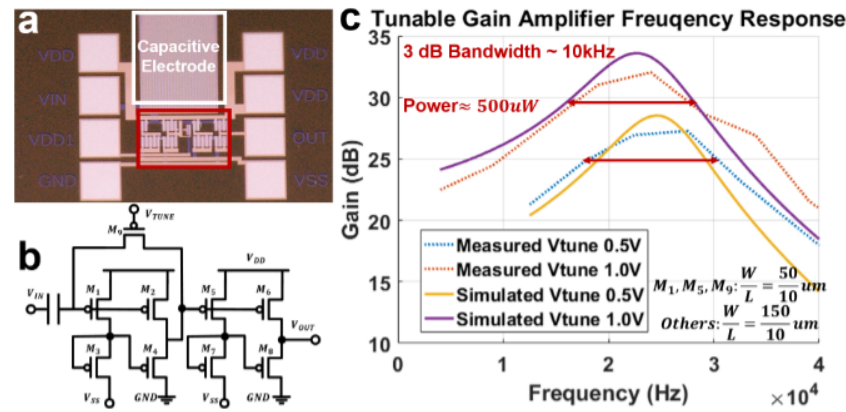

Figure 4: $a \& b$. Die photo and schematic of the ultra-thin $a c$ tive electrode prototype; $\mathrm{c}$. measured frequency responses of the amplifier and simulated results based on the device's compact model. $\mathrm{V}_{\mathrm{DD}}=3 \mathrm{~V}, \mathrm{~V}_{\mathrm{SS}}=-3 \mathrm{~V}$.

it's $3 \mathrm{~dB}$ bandwidth is less than $20 \mathrm{~Hz}$, which cannot meet the performance requirement of most bio-signal applications.

Here, for the first time, we report an ultra-thin active electrode integrated with a CNT-TFT based amplifier, which achieves both an ultra-thin form factor and a bandwidth greater than $10 \mathrm{kHz}$ running at $20 \mathrm{kHz}$. In contrast to [17], which required manual efforts to wire electrodes with the amplifier, we integrated everything in one ultrathin substrate. Unlike silicon circuits, most flexible TFT circuits operate $\sim 1-100 \mathrm{kHz}$ due to the limited temperature tolerance of the ultra-thin substrate $[7,20]$. Thus, in comparison with the state-ofthe-art flexible circuits [17-19], the performance of our fabricated CNT-TFT amplifier is highly competitive. Please note that $\sim \mathrm{GHz}$ performance has been reported for CNT based nanometer devices using high temperature silicon process on a rigid substrate, which is completely different from CNT-TFT devices fabricated on a flexible substrate.

The die photo and schematic of the fabricated active electrode is shown in Fig. 4a-b. The total area of the active electrode is only ( $\sim 300 \times 650 \mu \mathrm{m}^{2}$ excluding pads), where the white block is the electrode/sensor and the red block is the two-stage CNT-TFT based amplifier using only p-type devices. The fabrication of stable n-type CNT-TFTs remains a longstanding challenge and the performance is much worse than the p-type devices [21,22], thus a mono-type design style, named Pseudo-CMOS [23], is used to design the twostage amplifier $\left(M_{1}-M_{8}\right)$. One additional feedback transistor $\left(M_{9}\right)$ is used to control the feedback path and to provide tunability for the frequency response.

As shown in Fig. 4c, adjusting $V_{\text {tune }}$ can tune the frequency response, which offers greater flexibility and support broader application scenarios for the proposed active electrode. The low-frequency attenuation is determined by the effective capacitance value of the electrode. And, the overall frequency response can be optimized by properly sizing the transistors and choosing a suitable dielectric material. In addition to achieving the state-of-the-art performance ( $\sim 32 \mathrm{~dB}$ gain and $\sim 10 \mathrm{kHz} 3 \mathrm{~dB}$ bandwidth), the peak power consumption of the active electrode is $\sim 500 \mu W$ only at a supply voltage of $3 \mathrm{~V}$, which makes sharing of the power supply with silicon circuits without power conversion feasible. A designated active electrode for the ECG application will be described in Sec. 3.

The demonstrated performance, size, power consumption, and conformability of the proposed ultra-thin active electrode show its great potentials for bio-medical applications, such as ECG, EMG, electroencephalogram (EEG) and electrooculogram (EOG) (which usually require a bandwidth $<2 \mathrm{kHz}$ [24]). There are several directions for further improvement of the amplifier design, which will be discussed in Sec. 5.

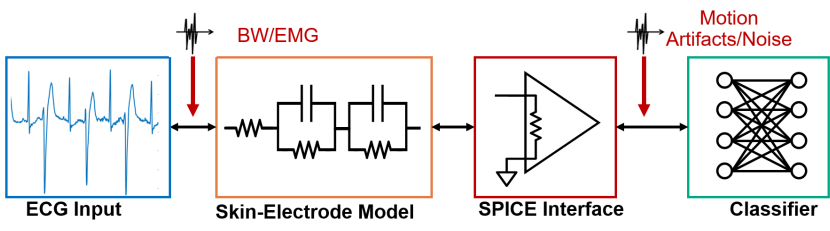

Figure 5: Developed end-to-end simulation framework, from emerging skin-sensor-silicon interface to machine learning classifier.

Table 1: Equivalent Parallel RC Model

\begin{tabular}{c|c|c|c|c}
\hline RC model & Skin & Electrode & Small Gap & Large Gap \\
\hline \hline$R(\Omega)$ & $10 \mathrm{~K}$ & $1 \mathrm{M}$ & $10 \mathrm{M}$ & $100 \mathrm{M}$ \\
\hline$C(F)$ & $\mathrm{N} / \mathrm{A}$ & $10 \mathrm{n}$ & $200 \mathrm{p}$ & $20 \mathrm{p}$ \\
\hline
\end{tabular}

\section{END-TO-END SIMULATION FRAMEWORK}

In this section, we introduce a simulation framework for systematic and quantitative analysis of an SSS link based on the active electrode. As shown in Fig. 5, the framework contains four main parts: the ECG inputs (including noise), the skin-electrode model, the SPICE model for CNT-TFT based amplifiers and the deep learning-based classifier for atrial fibrillation (AF) detection. We built this framework in MATLAB with customized interfaces to the SPICE engine and the AF classifier. This framework, enabling us to systematically explore and analyze how front-end skin-sensor interface will affect the overall classification accuracy, will be open-sourced to enable further innovations on skin electronics based on active electrodes. In the following, we introduce the setup for each of the four parts.

\subsection{ECG and Noise Database}

Experiments in this study use ECG signals from the PhysioNet MIT$\mathrm{BIH}$ database for performance analysis [16]. A typical waveform and spectrum of the MIT-BIH sample is shown in Fig. 6a-b. Later, we will use the AF detection for ECG as a driving application to evaluate the improvement achieved by the active electrode. Critical information for AF detection lies within $0.67-150 \mathrm{~Hz}$, which is an important guideline the amplifier design [25, 26].

Noise injection: To simulate the noisy ECG signals, noises from the MIT-BIH noise stress database [15] are added to the clean ECG signal described as following:

$$
\begin{aligned}
& \text { Noisy ECG = Clean ECG }+\alpha * \text { Noise } \\
& S N R=10 \log \left(S_{\text {power }} /\left(N_{\text {power }} * \alpha^{2}\right)\right)
\end{aligned}
$$

where $S_{\text {power }}$ and $N_{\text {power }}$ stand for the signal power and the noise power respectively and $\alpha$ is a scale factor. Based on Eqs. (1)-(2), we can generate noisy ECG signals with various SNR levels.

\subsection{Skin-electrode Equivalent Model}

The skin-electrode contact can be modeled as series of parallel RC circuits, where each coupling layer, such as the skin, the electrode dielectric and the air gap, can be modeled by a RC equivalent circuit and such a simple RC approximation turns out to be sufficiently accurate for analyzing low frequency bio-medical signals $(<2 \mathrm{kHz})$ [14]. Typical RC values for the skin, the electrode dielectric and air gaps are summarized in Table 1.

\subsection{Ultra-thin CNT-TFT Based Amplifier}

We used the recently developed SPICE model for the CNT-TFT [20], which has been thoroughly validated with wafer level CNT-TFT devices and circuits, for simulation and optimization of the amplifier. 

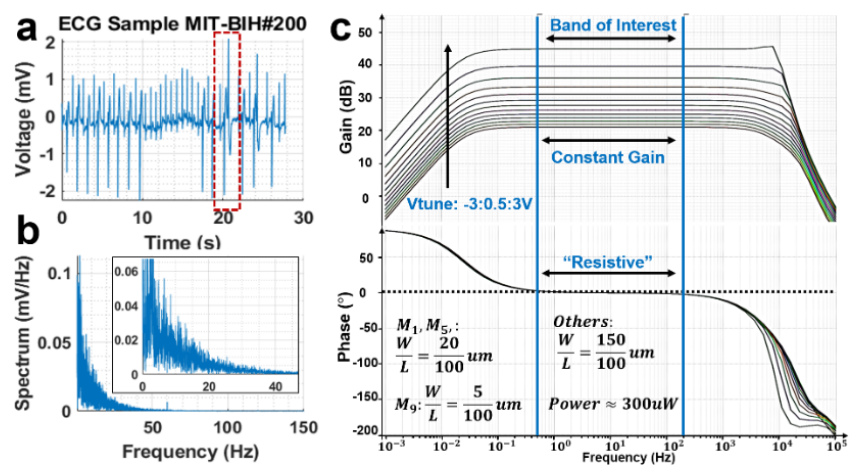

Figure 6: a\&b. Recording of a MIT-BIH ECG signal; c. designed amplifier for ECG applications.

We extracted all model parameters based on our CNT-TFT's measurements and validated the results with measurement from the fabricated amplifier as shown in Fig. 4c, where simulation results match well with the measured frequency responses. After confirming strong correlation between simulation and measurement results, the CNT-TFT model is used for design and optimization of the amplifier. Specifically, we follow the same topology described in Fig. 4b and optimize transistor sizes for AF detection from the ECG signals. Using long-channel $(100 \mu \mathrm{m})$ devices and optimized CNT-TFT ratios, the amplifier achieves great amplification $(>30 \mathrm{~dB}$ when $\left.V_{\text {tune }}>2 \mathrm{~V}\right)$ meanwhile no attenuation is introduced $\left(\sim 0^{\circ}\right.$ phase shift) in the band of $0.67-150 \mathrm{~Hz}$, which is well-suited for ECG signals as shown in Fig. 6c.

\subsection{Atrial Fibrillation Classifiers}

Electrocardiogram (ECG) recording is an important clinical tool for detecting cardiac disorders. Among them, atrial fibrillation (AF) is the most prevalent cardiac arrhythmia and can occur in sustained or intermittent episodes [25]. We benchmarked a feature-based and a deep learning approach on single-lead ECG recordings for the task of AF detection. The feature-based approach extracts various hand-crafted features on time/frequency domain, including heart rate variability (HRV) and morphological characteristics [26], and then feed them to a random forest for classification. However, as shown in Fig. 10a the change of rhythm from normal to AF ECGs has high variations, and such generic features may not be sufficient to fully represent the underlying characteristic of ECGs. Against this backdrop, we evaluated deep learning-based approach to automatically learn features at multiple levels of abstraction. Specifically, we deployed ResNet [27] as the network structure, of which the convolutional layer is the major feature learning component. The network takes fixed-length segments of 5 seconds each as input and produces a prediction for each segment. The overall classification for an entire ECG signal is the average of individual, segment-wise predictions. These two classifiers are used to quantify the impact of the front-end SSS design on the system level classification accuracy.

\section{SIMULATION RESULTS AND ANALYSIS}

With our simulation framework, we conducted both frequency and time domain analysis, which provides comprehensive evaluation. First, we analyzed the benefits brought by the conformable contact, which essentially eliminates the attenuation caused by air gaps. We then examined the SNR boosts achieved by the active electrode. We also made comparisons with advanced signal processing methods. Finally, we investigated the impact of the input ECG's SNR on the accuracy of the AF classification.

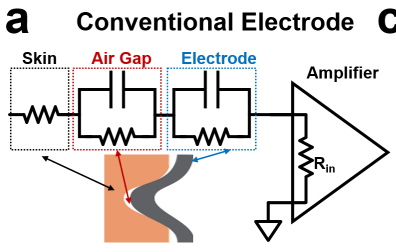

b Ultra-thin Electrode
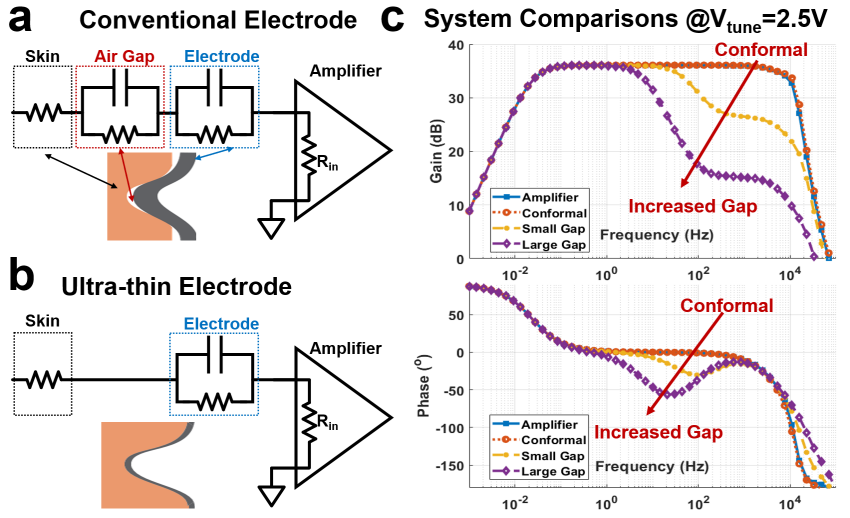

Figure 7: Attenuations in the frequency domain.

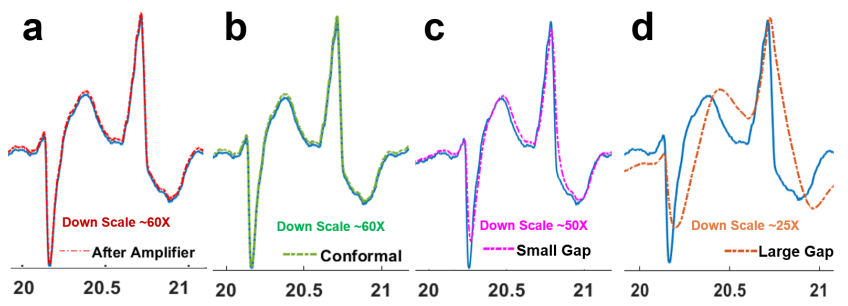

Figure 8: Attenuations in the time domain.

\subsection{Ultra-thin Electrode Enabled Attenuation Reduction}

The equivalent circuit diagrams of a traditional electrode and an ultra-thin electrode are shown in Fig. 7a-b, where the air gap is eliminated by a conformable contact of the ultra-thin electrode. Based on the RC parameters in Table 1, we simulated the frequency response of the skin-sensor-amplifier interfacing with a conformable contact, a small air gap and a big air gap respectively and summarized the responses in Fig. 7c. We can observe that the unavoidable air gap of a traditional electrode introduces attenuations in both gain and phase of the skin-electrode-amplifier link. For the time domain analysis, we chose an ECG segment with sharp dynamic behaviors, indicated by the red block in Fig. 6a, which has rich information in the frequency domain. For better visualization, we scale the amplitude of the output waveform and overlap it with the input waveform. As shown in Fig. 8, there are significant discrepancies and attenuations caused by the air gap while the conformable electrode shows great consistency between the input and output waveforms. For traditional electrodes, the air gap varies and the motion changes lead to unpredictable attenuations and noises, which pose significant challenges for AF detection. On the other hand, ultra-thin electrode minimizes the motion induced noises as the air gaps are eliminated.

\subsection{Active Electrode Enabled SNR Boosts}

In this following, we analyze the improvement to signal quality due to the active electrode under the motion artifacts, the BW noise and the EMG noise. Locations to inject these noises in an SSS link are indicated in the Fig. 5. Since the motion noises are suppressed for the ultra-thin active electrode, we assume that the motion noise mainly impacts the interconnects between flexible electrode and rigid silicon chip. EMG and BW noises, caused by body potential changes, are thus mixed with the ECG signal at the origin of the 

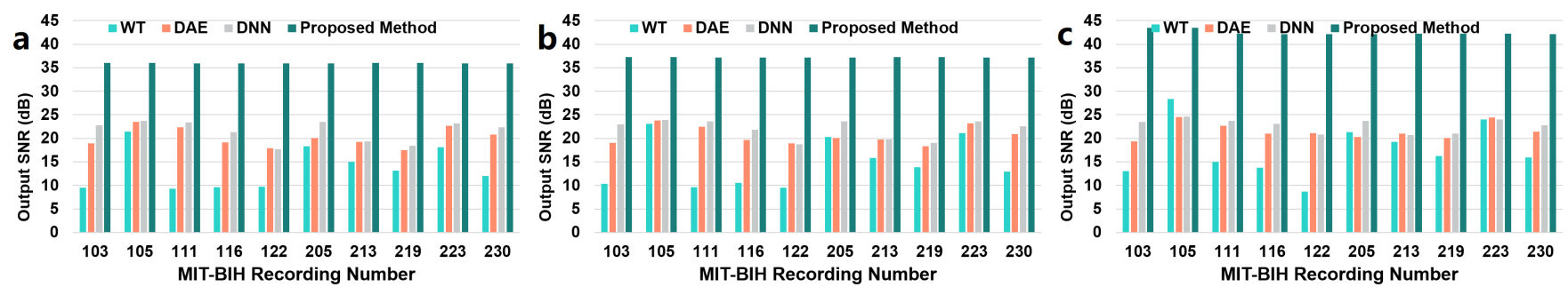

Figure 9: Simulated output signal's SNR under different motion noise levels: a. $\mathrm{SNR}=0 \mathrm{~dB} ; \mathrm{b}$. $\mathrm{SNR}=1.25 \mathrm{~dB}$; c. $\mathrm{SNR}=5 \mathrm{~dB}$.

Table 2: Evaluations under BW and EMG noises.

\begin{tabular}{c|l|l|l|l|l|l|l|l|l|l|l|l}
\hline $\begin{array}{c}\text { BW } \\
\text { Noise }\end{array}$ & MIT-BIH \# & 103 & 105 & 111 & 116 & 122 & 205 & 213 & 219 & 223 & 230 & Average \\
\hline $\begin{array}{c}\text { Input } \\
\text { SNR }\end{array}$ & 0.00 & 2.66 & 2.68 & 2.64 & 2.56 & 2.55 & 2.57 & 2.67 & 2.65 & 2.63 & 2.61 & 2.616 \\
\cline { 2 - 12 } & 1.25 & 3.91 & 3.93 & 3.89 & 3.81 & 3.80 & 3.82 & 3.92 & 3.90 & 3.88 & 3.86 & 3.866 \\
\cline { 2 - 11 } & 5.00 & 7.66 & 7.68 & 7.64 & 7.56 & 7.55 & 7.57 & 7.67 & 7.65 & 7.63 & 7.61 & 7.616 \\
\hline
\end{tabular}

SSS link. In principle, the proposed method should be able to effectively overcome the motion noise while no significant difference would be observed for BW and EMG noises. We examine the SNR improvement under the motion noise first followed by evaluation with EMG and BW noises.

Comparison under the motion noise: We compared the SNR improvement achieved by our proposed method with those purely based on signal processing methods, such as wavelet transform (WT), denoising auto-encoder (DAE) and deep neural network (DNN) methods [10,11]. For a fair comparison, the same 10 PhysioNet ECG recordings are chosen for the analysis and three different input SNR levels, $5 \mathrm{~dB}, 1.25 \mathrm{~dB}$ and $0 \mathrm{~dB}$ respectively, are generated based on Eqs. (1)-(2) for simulation. The simulation results are summarized in Fig. 9, where $\mathrm{x}$-axis is the ECG recording index and $\mathrm{y}$-axis is the output signal's SNR. The ultra-thin active electrode clearly outperforms all state-of-the-art signal processing methods for motion suppression under a wide range of noise levels. The peak power consumption of the active electrode is only about 300 $\mu W$, which shows significant energy saving compared to the heavy signal processing methods $(>10 \mathrm{~mW})$. Note that our method is a hardware-based solution which can be combined with advanced signal processing to further improve the system performance.

Evaluations under BW and EMG noises: Similarly, evaluation results of the active electrode under EMG and BW noises are summarized in Table 2, where the first row indicates the recording index and following rows correspond to the output SNR under different input noise level. Not surprisingly, the active electrode slightly suppresses the BW noise (mainly lies in $<0.5 \mathrm{~Hz}$ ) since the amplifier has a $3 \mathrm{~dB}$ corner $\sim 0.1 \mathrm{~Hz}$ as shown in Fig. 6c, which partially filters out the BW noise. For the EMG noise, the signal quality drops slightly $(\sim 3 \mathrm{~dB})$ since the widespread nature (over $0-500 \mathrm{~Hz}$ ), which has a large overlap with the amplifier's high gain region. The slightly SNR drop is acceptable since the motion noise is the most significant noise source (Motion : EMG : BW $\approx 10: 1: 1$ ), as presented in Fig. $2 b$.

\subsection{SNR vs. Classification Accuracy}

To assess the impact of the front-end SSS interfacing on the system level accuracy of AF detection, we evaluated both feature-based and deep learning-based AF classifiers under various motion noise levels (SNR ranging from $0 \mathrm{~dB}$ to $35 \mathrm{~dB}$ ) on publicly available PhysioNet data set, which contains 8,528 ECG recordings [24]. Five-fold cross-validation is applied to assess the two classifiers, and the

\begin{tabular}{r|l|l|l|l|l|l|l|l|l|l|l|l}
\hline $\begin{array}{r}\text { EMG } \\
\text { Noise }\end{array}$ & MIT-BIH \# & 103 & 105 & 111 & 116 & 122 & 205 & 213 & 219 & 223 & 230 & Average \\
\hline $\begin{array}{r}\text { Input } \\
\text { SNR }\end{array}$ & 0.00 & -3.05 & -3.03 & -3.06 & -3.15 & -3.15 & -3.14 & -3.04 & -3.06 & -3.08 & -3.10 & -3.086 \\
\cline { 2 - 12 } & 1.25 & -1.80 & -1.78 & -1.81 & -1.90 & -1.90 & -1.89 & -1.79 & -1.81 & -1.83 & -1.85 & -1.836 \\
\cline { 2 - 12 } & 5.00 & 1.95 & 1.97 & 1.94 & 1.85 & 1.85 & 1.86 & 1.96 & 1.94 & 1.92 & 1.90 & 1.914 \\
\hline
\end{tabular}
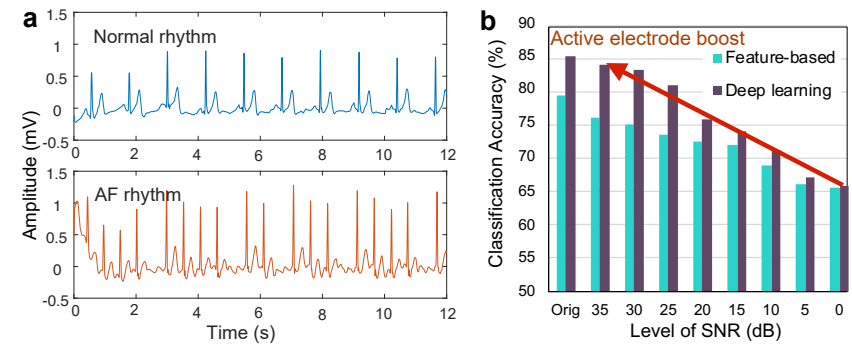

Figure 10: a. The comparison between normal and AF ECG recordings; $b$. classification accuracy improvement with increasing level of SNR on both of feature-based and deep learning-based approach.

classification accuracy is measured using the averaged true positive rates over normal and AF ECG recordings. The ResNet model is trained with error backpropagation using Adam optimizer and categorical cross-entropy as the loss function. During training, we reduce the learning rate by a factor of 10 until validation loss converges. The weights that achieve the best validation accuracy are selected for final evaluation. As shown in Fig. 10b, the accuracy of both the hand-crafted classifier and the deep neural network classifier increases as the SNR improves. Utilizing our front-end SSS design, we can boost the SNR from $0 \mathrm{~dB}$ to $>35 \mathrm{~dB}$, which in turn improves classification accuracy by $>11 \%$ and $>19 \%$ for the handcrafted classifier and the deep-learning based classifier respectively. The ultra-thin SSS interface can alleviate signal attenuations and boost the signal quality under motion noises, which is critical to achieving high accuracy for AF classification.

\section{DISCUSSION AND FUTURE WORK}

One main challenge for the ultra-thin TFT circuits is that TFTs exhibit high $1 / \mathrm{f}$ noise, whose magnitude decreses as the freuency increses, shown in Fig. 11. From the measured 1/f noise over 0-100 $\mathrm{kHz}$, we can see that the $1 / \mathrm{f}$ noise falls directly in the band of interest for ECG signals. The chopper stabilization technique can be used to effectively reduce the $1 / \mathrm{f}$ noise [28]. The key idea is using a carrier signal to up modulate the ECG signals to a relative high frequency range, where the $1 / \mathrm{f}$ noise is significantly lower $\left(\sim 10^{4} \mathrm{X}\right)$ than the low frequency range as illustrated in Fig. 11. Our fabricated CNTTFT amplifier has already demonstrated an operation speed $>20 \mathrm{kHz}$, 


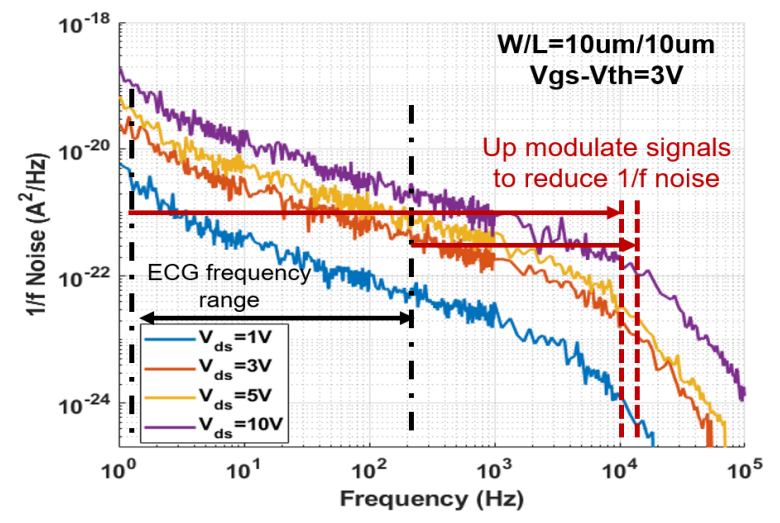

Figure 11: Measured 1/f Noise for a TFT transistor.

thus this technique can be readily applicable for reducing $1 / \mathrm{f}$ noise [28]. A single end topology is currently used for our prototyped amplifier; however, for better suppression of common mode noise, a differential topology should be considered. Other system aspects, such as hardware/software co-design and exploration of trade-offs between the silicon electronics and the skin electronics, could also be investigated.

\section{CONCLUSION}

In this paper, we introduce an emerging paradigm for skin electronics, which shows great potential for high quality and long-term skin-sensor-silicon interfacing. Specifically, we propose an active electrode design and demonstrate a prototype of skin electronics based SSS interfacing. Besides measurement results, quantitative and systematic simulation results further confirm that the ultrathin SSS interfacing can bring significant accuracy boosts for AF detection. In addition to the driving application ( $\mathrm{AF}$ classification for ECG signals), the developed active electrode and simulation framework can be applied to other applications involving humanmachine interfacing. Finally, promising future research directions are highlighted for further innovations on skin electronics.

\section{ACKNOWLEDGMENTS}

This material is based upon work supported, in part, by Air Force Research Laboratory under agreement number FA8650-15-2-5401. The U.S. Government is authorized to reproduce and distribute reprints for Governmental purposes notwithstanding any copyright notation thereon. The views and conclusions contained herein are those of the authors and should not be interpreted as necessarily representing the official policies or endorsements, either expressed or implied, of Air Force Research Laboratory or the U.S. Government. The authors would like to thank Dr. Rongsheng Chen and Pro. Yuan Liu for providing noise measurements and vital suggestions.

\section{REFERENCES}

[1] Bryant Chu, William Burnett, Jong Won Chung, and Zhenan Bao. Bring on the bodynet. Nature News, 549(7672):328, 2017.

[2] Mallory L Hammock, Alex Chortos, Benjamin C-K Tee, Jeffrey B-H Tok, and Zhenan Bao. 25th anniversary article: the evolution of electronic skin (e-skin): a brief history, design considerations, and recent progress. Advanced materials, 25 (42):5997-6038, 2013.

[3] Takao Someya, Zhenan Bao, and George G Malliaras. The rise of plastic bioelec tronics. Nature, 540(7633):379, 2016.

[4] Jae Woong Jeong, Woon Hong Yeo, Aadeel Akhtar, James J.S. Norton, Young Jin Kwack, Shuo Li, Sung Young Jung, Yewang Su, Woosik Lee, Jing Xia, Huanyu Cheng, Yonggang Huang, Woon Seop Choi, Timothy Bretl, and John A. Rogers. Materials and optimized designs for human-machine interfaces via epidermal electronics. Advanced Materials, 25(47):6839-6846, 2013.
[5] Jae Woong Jeong, Min Ku Kim, Huanyu Cheng, Woon Hong Yeo, Xian Huang, Yuhao Liu, Yihui Zhang, Yonggang Huang, and John A. Rogers. Capacitive epidermal electronics for electrically safe, long-term electrophysiological measurements. Advanced Healthcare Materials, 3(5):642-648, 2014.

[6] Ting Lei, Xiyuan Chen, Gregory Pitner, H-S Philip Wong, and Zhenan Bao. Removable and recyclable conjugated polymers for highly selective and highyield dispersion and release of low-cost carbon nanotubes. Fournal of the American Chemical Society, 138(3):802-805, 2016

[7] Tsung-Ching Huang, Leilai Shao, Ting Lei, Raymond Beausoleil, Zhenan Bao, and Kwang-Ting Cheng. Robust design and design automation for flexible hybrid electronics. In Circuits and Systems (ISCAS), 2017 IEEE International Symposium on, pages 1-4. IEEE, 2017.

[8] Jin Young Oh, Simon Rondeau-Gagné, Yu-Cheng Chiu, Alex Chortos, Franziska Lissel, Ging-Ji Nathan Wang, Bob C Schroeder, Tadanori Kurosawa, Jeffrey Lopez, Toru Katsumata, et al. Intrinsically stretchable and healable semiconducting polymer for organic transistors. Nature, 539(7629):411, 2016.

[9] Alex Chortos, Ghada I Koleilat, Raphael Pfattner, Desheng Kong, Pei Lin, Roda Nur, Ting Lei, Huiliang Wang, Nan Liu, Ying-Chih Lai, et al. Mechanically durable and highly stretchable transistors employing carbon nanotube semiconductor and electrodes. Advanced Materials, 28(22):4441-4448, 2016.

[10] Manas Rakshit and Susmita Das. An efficient ECG denoising methodology using empirical mode decomposition and adaptive switching mean filter. Biomedical Signal Processing and Control, 40:140-148, feb 2018. ISSN 17468094.

[11] Peng Xiong, Hongrui Wang, Ming Liu, Suiping Zhou, Zengguang Hou, and Xiuling Liu. ECG signal enhancement based on improved denoising auto-encoder. Engineering Applications of Artificial Intelligence, 52:194-202, 2016.

[12] Nick Van Helleputte, Sunyoung Kim, Hyejung Kim, Jong Pal Kim, Chris Van Hoof, and Refet Firat Yazicioglu. A $160 \mu$ a biopotential acquisition asic with fully integrated ia and motion-artifact suppression. In Solid-State Circuits Conference Digest of Technical Papers (ISSCC), 2012 IEEE International, pages 118-120. IEEE, 2012.

[13] Martin J Burke and Denis T Gleeson. A micropower dry-electrode ecg preamplifier. IEEE Transactions on Biomedical Engineering, 47(2):155-162, 2000.

[14] Yu Mike Chi, Tzyy-Ping Jung, and Gert Cauwenberghs. Dry-contact and noncontact biopotential electrodes: Methodological review. IEEE reviews in biomedical engineering, 3:106-119, 2010.

[15] George B Moody, W Muldrow, and Roger G Mark. A noise stress test for arrhythmia detectors. Computers in cardiology, 11(3):381-384, 1984.

[16] George B Moody and Roger G Mark. The impact of the mit-bih arrhythmia database. IEEE Engineering in Medicine and Biology Magazine, 20(3):45-50, 2001.

[17] Tsuyoshi Sekitani, Tomoyuki Yokota, Kazunori Kuribara, Martin Kaltenbrunner, Takanori Fukushima, Yusuke Inoue, Masaki Sekino, Takashi Isoyama, Yusuke Abe, Hiroshi Onodera, and Takao Someya. Ultraflexible organic amplifier with biocompatible gel electrodes. Nature Communications, 7, 2016.

[18] Tiffany Moy, Liechao Huang, Warren Rieutort-Louis, Can Wu, Paul Cuff, Sigurd Wagner, James C. Sturm, and Naveen Verma. An EEG Acquisition and BiomarkerExtraction System Using Low-Noise-Amplifier and Compressive-Sensing Circuits Based on Flexible, Thin-Film Electronics. IEEE fournal of Solid-State Circuits, 52 (1):309-321, 2017.

[19] Rei Shiwaku, Hiroyuki Matsui, Kuniaki Nagamine, Mayu Uematsu, Taisei Mano, Yuki Maruyama, Ayako Nomura, Kazuhiko Tsuchiya, Kazuma Hayasaka, Yasunori Takeda, Takashi Fukuda, Daisuke Kumaki, and Shizuo Tokito. A printed organic amplification system for wearable potentiometric electrochemical sensors. Scientific Reports, 8(1):1-8, 2018. ISSN 20452322.

[20] Leilai Shao, Tsung-Ching Huang, Ting Lei, Zhenan Bao, Raymond Beausoleil, and Kwang-Ting Cheng. Compact modeling of carbon nanotube thin film transistors for flexible circuit design. In Design, Automation \& Test in Europe Conference \& Exhibition (DATE), 2018, pages 491-496. IEEE, 2018.

[21] Huiliang Wang, Peng Wei, Yaoxuan Li, Jeff Han, Hye Ryoung Lee, Benjamin D Naab, Nan Liu, Chenggong Wang, Eric Adijanto, Benjamin C-K Tee, et al. Tuning the threshold voltage of carbon nanotube transistors by n-type molecular doping for robust and flexible complementary circuits. Proceedings of the National Academy of Sciences, 111(13):4776-4781, 2014.

[22] Le Cai and Chuan Wang. Carbon nanotube flexible and stretchable electronics. Nanoscale research letters, 10(1):1, 2015.

[23] Tsung-Ching Huang, Kenjiro Fukuda, Takao Someya, and Kwang-Ting Cheng. Pseudo-cmos: A design style for low-cost and robust flexible electronics. IEEE Transactions on Electron Devices, 58(1):141-150, 2011.

[24] Gari D Clifford, Chengyu Liu, Benjamin Moody, Li-wei H Lehman, Ikaro Silva, Qiao Li, AE Johnson, and Roger G Mark. Af classification from a short single lead ecg recording: The physionet computing in cardiology challenge 2017. Proceedings of Computing in Cardiology, 44:1, 2017.

[25] Fernando Andreotti, Oliver Carr, Marco AF Pimentel, Adam Mahdi, and Maarten De Vos. Comparing feature-based classifiers and convolutional neural networks to detect arrhythmia from short segments of ecg. Computing, 2017.

[26] Vykintas Maknickas, Algirdas Maknickas, and LLC Tesonet. Atrial fibrillation classification using qrs complex features and lstm. Computing, 2017.

[27] Kaiming He, Xiangyu Zhang, Shaoqing Ren, and Jian Sun. Deep residual learning for image recognition. In Proceedings of the IEEE conference on computer vision and pattern recognition, pages 770-778, 2016.

[28] Op-Amp Imperfections. Circuit techniques for reducing the effects of op-amp imperfections: autozeroing, correlated double sampling, and chopper stabilization. Proceedings of the IEEE, 84(11), 1996. 